\title{
sciendo
}

10.2478/AMB-2019-0020

\section{RENAL CHANGES IN COCAINE ABUSE AND ADDICTION}

\author{
M. Nikolova', VI. Milenova', D. Yosifov', Y. Vlahov'², V. Tenev \\ ${ }^{1}$ Medical University - Sofia, Bulgaria \\ ${ }^{2}$ Medical Faculty, Sofia University Sv. Kl. Ohridski - Sofia, Bulgaria \\ ${ }^{3}$ University Hospital, University of lowa - lowa city, IA, USA
}

\begin{abstract}
Cocaine is a natural alkaloid extracted from the leaves of the South American plant Erythroxylum coca or synthesized chemically. After cannabis, it is the second most frequently abused recreational substance worldwide. Cocaine can affect every tissue and organ within the human body, including the kidneys, causing tissue ischemia due to vasoconstriction, endothelial dysfunction and damage, procoagulant activity and oxidative stress with subsequent ischemic infarctions and fibrosis. The renal changes in cocaine abuse and addiction are due to rhabdomyolysis, ischemic, hypertensive, and inflammatory changes with the development of cell proliferation and fibrosis. The authors present three patients with cocaine-associated renal damage and discuss the underlying mechanisms of cocaine-induces tissue changes.
\end{abstract}

Key words: cocaine, systemic effects, renal damage, rhabdomyolysis, ischemia, hypertension, coagulation, cholesterol

Corresponding author: Milena Nikolova, MD, PhD, Clinic of Nephrology, University Hospital Alexandrovska, Medical University - Sofia, 1 Sv. G. Sofiyski Str. 1431, Bulgaria, e-mail: milena_i_dani@abv.bg

\section{INTRODUCTION}

$\mathrm{C}$ ocaine is a natural alkaloid extracted from the leaves of the South American plant Erythroxylum coca or synthesized chemically. After cannabis, it is the second most frequently abused recreational substance worldwide - between 14 and 21 million people are estimated to have used this drug every year [1].

Cocaine has been used by the indigenous South American tribes for stimulant, anesthetic, religious and recreational purposes in the form of coca plant (Erythroxylum coca) leaves - chewed or processed to extract the active alkaloid [2]. In 1855 Friedrich Gaedcke was the first who isolated the alkaloid and named it erythroxyline, and in 1898 Richard Willstätter synthesized it from tropinone. As a drug, cocaine was initially used as a painkiller and subsequently as local anesthetic with unique vasoconstrictive properties. In 1879 it was introduced for the treatment of morphine addiction, and subsequently - as a psychostimulant and appetite-suppressing agent. Later, cocaine was referred to as a controlled substance due to its addictive potential. Gradually, cocaine became the second most abused illicit drug used by all socioeconomic strata, age, and demographic, economic, social, political, and religious groups all over the world. It can be insufflated (snorted), taken orally (gingival route and chewing coca leaves), smoked, administered rectally, and injected intravenously or intramuscularly, both alone and in combination with 
heroin (speedball) [3]. Nowadays, its use is limited as local/topical anesthetic, mainly in ophthalmology.

From a pharmacological point of view, cocaine has sympathomimetic effects, influencing also serotonin, kappa-opioid, sigma, D1, and NMDA receptors and membrane ion transport. Moreover, it has long-term endocrine, immunological and genetic effects.

The pharmacodynamic effects of cocaine are determined by its three major actions [1, 2, 4]: increased release of catecholamines in the synaptic cleft (via stabilization of the dopamine transporter), decreased mediator reuptake (via blockage of the presynaptic dopamine transporter), and blockage of neuronal membrane sodium channels (producing local anesthetic effect). The appetite-suppressing and locomotor effects of cocaine are explained by its interaction with serotonin 5-HT3 and 5-HT2 receptors, and changes with dopamine levels in the substantia nigra.

The net effect of the ligand-receptor interactions of cocaine is sympathomimetic effect action with buildup of dopamine in the limbic system structures (mainly nucleus accumbens) and stimulation of pleasure and reward feeling. The latter can explain the addiction and dependence in long-term cocaine abuse [5]. Cocaine can also increase dopamine levels in other brain areas, linked to the limbic system, including memory centers (hippocampus and amygdala) and the frontal cortex. It has been suggested that the repeated exposure to cocaine (with increase in dopamine levels in the hippocampus and amygdale) leads to functional and organic changes producing the characteristic compulsory craving for repeated intake due to the persistent memory of cocaine use in the past [6]. Moreover, the repeated increase in dopamine levels in the frontal cortex in cocaine abuse leads to functional and structural changes with decreased inhibition of the urges generated in the nucleus ac- cumbens, hippocampus, and amygdala and subsequent addictive pattern. As it was mentioned above, the interactions with serotonin receptors may explain the mood changes and the appetite-suppressing effects of cocaine.

Another molecular effect that can explain the addiction in cocaine intake is the genetic impact of this alkaloid - alteration in the amount of dopamine transporters and dopamine receptors on nerve cells via modulation of gene expression (genetic, transcriptional and epigenetic effects of cocaine, mediated by $\triangle$ FosB) and neuropasticity $[5,7]$. These very longterm effects due to genetic and epigenetic changes in the brain make cocaine addiction very difficult to counteract. A serious long-term effect of cocaine is dopamine depletion of the brain, probably responsible for the withdrawal symptoms [4].

Cocaine has multiple extracranial systemic effects due to its vasoconstrictive, prothrombotic, endotheliotoxic and arrhythmogenic properties, potentially affecting every organ in the human body, including vascular endothelium, immune and endocrine systems $[1,2,8,9,10,11,12,13,14,15]$. The main extracranial effects of cocaine include:

- Arterial hypertension and malignant hypertension,

- Prothrombotic effect with increased thrombotic risk,

- Ischemic organ infarctions,

- Endothelial injury and vasculitis,

- Rhabdomyolysis,

- Acute kidney injury and chronic kidney disease.

Cocaine and its derivates and metabolites can affect the kidney, causing both acute and chronic renal injury (table 1). The major underlying mechanisms include: renal ischemia due to vasoconstriction and

Table 1. Renal changes in chronic cocaine abuse and addiction $[8,9,10,11,12,14,15]$

\begin{tabular}{|l|l|}
\hline Acute renal changes & Chronic renal changes \\
\hline Rhabdomyolysis and acute myoglobinuric renal failure & $\begin{array}{l}\text { Repetitive episodes of rhabdomyolysis with tubulo-intersti- } \\
\text { tial fibrosis }\end{array}$ \\
\hline $\begin{array}{l}\text { Hypertensive crisis and renal scleroderma crisis } \\
\text { Malignant hypertension with arteriolosclerosis } \\
\text { Hemolytic uremic syndrome } \\
\text { Disseminated intravascular coagulation } \\
\text { Necrotizing vasculitis }\end{array}$ & Chronic hypertensive nephropathy \\
Renal infarction & Accelerated atherosclerosis \\
\hline Acute tubulo-interstitial nephritis & Chronic ischemic nephropathy \\
\hline $\begin{array}{l}\text { Glomerular lesions: necrotizing, membranoproliferative, mesangioproliferative IgA glomerulonephritis, hepatitis- and HIV-associated } \\
\text { nephromathy, renal amyloidosis }\end{array}$ \\
\hline In utero exposure - urogenital, cardiovascular and nervous system abnormalities of the fetus. \\
\hline
\end{tabular}


activation of thrombogenesis, endothelial toxicity and vasculitis, rhabdomyolysis, tubulo-interstitial toxicity, stimulation of mesangial matrix synthesis and mesangial cell proliferation and fibrogenesis, inhibition of macrophageal IL-6 and IL-8 synthesis, changes in $\mathrm{T}$ - and B-cell immune response, development of infection-associated amyloidosis [8, 9, 10, 12, 13]. Moreover, cocaine exposure in utero may lead to urogenital, cardiovascular, nervous system and other abnormalities $[11,12,14,15]$. The most common types of renal injury are rhabdomyolysis-associated, hypertensive and ischemic changes (renal infarction and fibrosis).

We would like to present three cases of cocaine-associated renal damage.

\section{CASE PRESENTATION}

\section{Patient 1}

In 2010, a 44-years old male Caucasian patient was admitted to the Clinic of nephrology for diagnostic evaluation of a newly-diagnosed renal failure. The patient had a 10 years history of diabetes, arterial hypertension and obesity, echocardiographic data for dilative cardiomyopathy with no coronorographic stigmata of coronary artery stenosis, and reported heavy cocaine abuse for the past 10 years. The clinicallaboratory investigations revealed mild anemia (hemoglobin levels of $119 \mathrm{~g} / \mathrm{l}, \mathrm{RBC} 4.04 \mathrm{~T} / \mathrm{l}$ ), urea 13.0 $\mathrm{mmol} / \mathrm{l}$, creatinine $264 \mathrm{mcmol} / \mathrm{l}$, total cholesterol 3.57 $\mathrm{mmol} / \mathrm{l}$, triglycerides $1.21 \mathrm{mmol} / \mathrm{l}$, normal levels of uric acid, potassium, sodium, calcium, phosphates, liver enzymes, urinary protein excretion of $2.8 \mathrm{~g} / 24$ hours. Abdominal ultrasound investigation (figure 1) demonstrated both kidneys normal in size (111-112 $\mathrm{mm}$ ) with parenchymal width of $20-21 \mathrm{~mm}$, increased parenchymal echogenicity with cortical and medullar calcifications.

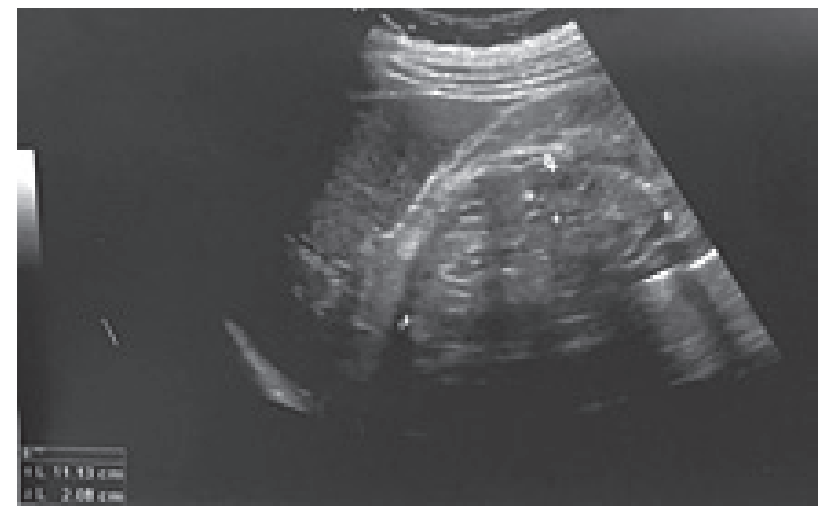

Fig. 1. Abdominal ultrasound of patient 1 , demonstrating diffuse renal parenchymal calcifications (cortical + medullary nephrocalcinosis)

\section{Patient 2}

A 23-years old male patient was admitted to the Clinic of Nephrology in 2001 for diagnostic evaluation of hematuria and low-grade proteinuria. The patient reported heavy heroin + cocaine abuse for the past 4 years. The clinical-laboratory investigations revealed normal whole blood count, creatinine, urea, total protein, albumin, liver enzymes, low total cholesterol 2.8 $\mathrm{mmol} / \mathrm{l}$, triglycerides $0.9 \mathrm{mmol} / \mathrm{l}$, proteinuria $0.4 \mathrm{~g} / 24$ h., erythrocyturia with low percentage of dysmorphic erythrocytes (12\%). The abdominal ultrasound revealed normal renal size, thinned parenchyma with increased echogenicity. The renal biopsy showed signs of chronic tubulo-interstitial nephritis with lymphocyte infiltration. The cessation of illicit drug abuse and a short-term corticosteroid course (prednisone $20 \mathrm{mg}$ a day for 30 days with reduction with $5 \mathrm{mg}$ every two weeks) lead to the disappearance of proteinuria and erythrocyturia.

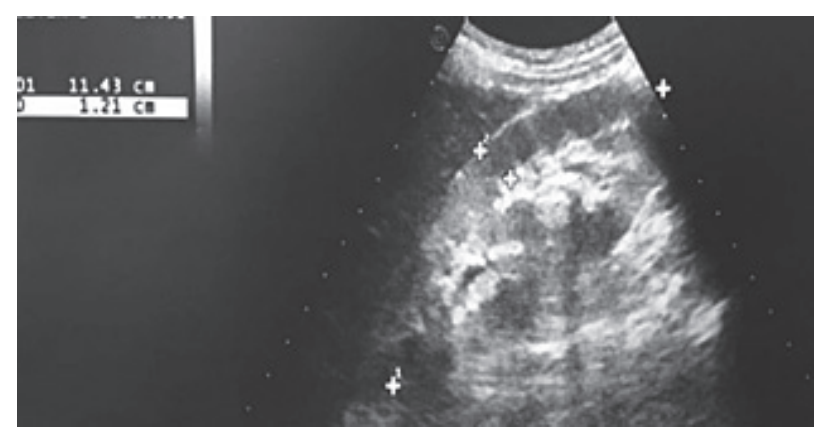

Fig. 2. Abdominal ultrasound of patient 2 - normal renal size, thinned parenchyma with increased echogenicity

\section{Patient 3}

A 36-years-old female patient was admitted to the clinic of Nephrology in 2016 for diagnostic evaluation of low-grade proteinuria, erythrocituria and renal failure. The patient reported recurrent urinary tract and throat infections, miscarriage in the past and incidental ("week-end party") cocaine use. The clinical-laboratory investigations revealed mild anemia (hemoglobin $108 \mathrm{~g} / \mathrm{l}, \mathrm{RBC} 3.9 \mathrm{~T} / \mathrm{l}$ ), urea $8.4 \mathrm{mmol} / \mathrm{l}$, creatinine $178 \mathrm{mcmol} / \mathrm{l}$, total cholesterol $4.03 \mathrm{mmol} / \mathrm{l}$, triglycerides $1.53 \mathrm{mmol} / \mathrm{l}$, normal sodium, potassium, calcium, phosphate, uric acid, total protein, liver enzymes, urinalysis revealed mass erythroocyturia nad low-grade proteinuria of $0.29 \mathrm{~g} / 24 \mathrm{~h}$. The abdominal ultrasound revealed both kidneys normal in size (111-114 mm), with parenchymal size $16-20 \mathrm{~mm}$ and marked increase in parenchymal echogenicity with prominent hypoechogenic pyramids, and cortical calcifications. Renal biopsy with 10 glomeruli showed expansion of matrix substance with mesangial cell 
proliferation and mesangial deposition of $\lg A, \lg$, C3, C3 deposition in arteriolar walls accompanied by focal interstitial fibrosis and lymphycytic infiltration, suggesting the presence of mesangioproliferative IgA glomerulonephritis and tubulo-interstitial nephritis. A short-term antibiotic course for urinary tract infection and low-dose corticosteroids (prednisone $30 \mathrm{mg}$ for 30 days with gradual decrease with $5 \mathrm{mg}$ every two weeks), along with cessation of cocaine use lead to decrease in serum creatinine to $130 \mathrm{mcmol} / \mathrm{l}$ and gradual increase in hemoglobin and RBC to the normal levels.

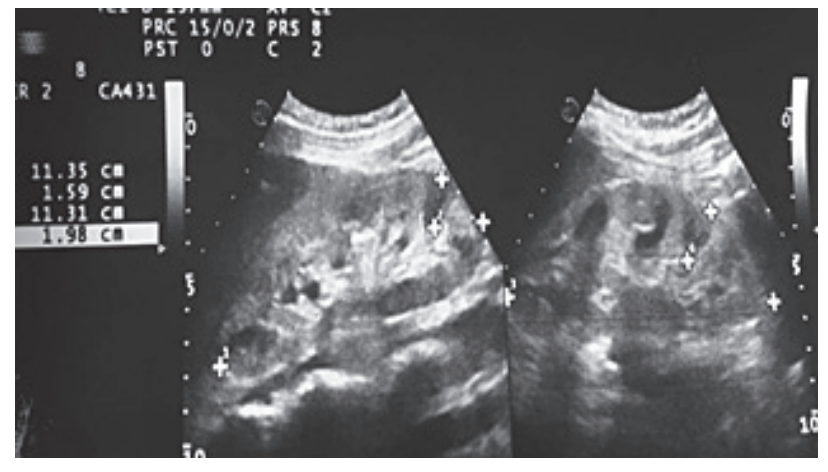

Fig. 3. Abdominal ultrasound of patient 3 -normal renal and parenchymal size and increased parenchymal echogenicity with prominent hypoechogenic, contrasting, pyramids, cortical calcifications

\section{DISCUSSION}

Cocaine is a sympathomimetic agent and its action is mediated by blockage of norepinephrine, dopamine and serotonin reuptake in the presynaptic nerve endings. It also blocks sodium channels (voltage-specific) and interacts with multiple neurotransmitters and their receptors in and outside the brain, kappa-opioid, sigma, D1, and NMDA receptors in the centra nervous system, on immune-competent, endothelial and other (mesenchymal) cells. Cocaine can affect every tissue and organ within the human body, including the kidneys, causing tissue ischemia due to vasoconstriction, endothelial dysfunction and damage, procoagulant activity and oxidative stress with subsequent ischemic infarctions and fibrosis, and my stimulating cellular receptors.

According to N Goel et al. [9], the cocaine-induced renal lesions develop via five major pathogenic pathways:

1. Sympathic activation with subsequent vasoconstriction (due to suppression of nitric oxide-mediated vasodilation);

2. Changes in endothelial function with increased production of endothelin and decreased synthesis of
NO by the endothelial cells - both due to direct effects upon the endothelium and changes in cytokine levels (i.e., inhibition of IL- 8 and IL-6 secretion with subsequent stimulation of TGF-beta secretion);

3. Development of procoagulant state due to platelet activation;

4. Inhibition of prostaglandin E2 and I2 (prostacyclin) secretion with subsequent vasoconstriction and platelet activation;

5. Generation of reactive oxygen species as by-products of cocaine degradation and inhibition of mitochondrial respiration, decrease of gluthatione levels and oxidation-mediated cell death.

Moreover, cocaine can alter immune function [13] due to the presence of dopamine-mediated alterations in B-, T- and NK-cell function, triggered by cocaine.

The net effects of these changes on the kidney are: development of renal ischemia, matrix accumulation, mesangial proliferation, inflammation and fibrosis. Some cocaine abusers develop renal amyloidosis depositions, probably as a result of the chronic infections, and hepatitis and HIV-associated renal glomerular changes.

An interesting finding in cocaine abusers and addicts is the low total cholesterol that seems to correlate with the risk of relapse after cocaine cessation [16].

The patients in our series show three different types of renal affection in cocaine use and addiction - the first has diffused mixed type nephrocalcinosis that can develop both due to ischemic nephropathy and repetitive episodes of rhabdomyolysis, the second has tubulo-interstitial nephritis that improves clinically after corticosteroid administration suggesting toxic and/or immune-mediated renal injury, and the third has both glomerular (mesangioproligerative IgA glomerulonephritis) and tubulo-interstitial involvement that also improve clinically after corticosteroid treatment. Two patients have low total cholesterol levels.

These cases suggest that cocaine abuse and addiction should be suspected in patients with both tubulointerstitial and glomerular diseases, especially in the presence of heart disease and low serum total cholesterol levels.

\section{REFERENCES}

1. Pomara C, Cassano T, D'Errico S et al. Data available on the extent of cocaine use and dependence: biochemistry, pharmacologic effects and global burden of disease of cocaine abusers. Cur Med Chem 2012;19(33):5647-5657. 
2. Gay GR, Inaba DS, Sheppard CW, Newmeyer JA. Cocaine: history, epidemiology, human pharmacology, and treatment. A perspective on a new debut for an old girl. Clin Toxicol 1975;8(2):149-178.

3. Hall AP, Henry JA. Illicit drugs and surgery. International Journal of Surgery 2007;5:365-370.

4. Quinn DI, Wodak A, Day RO. Pharmacokinetic and pharmacodynamic principles of illicit drug use and treatment of illicit drug users. Clin Pharmacokinet 1997;33(5):344-400.

5. Nestler EJ. The neurobiology of cocaine addiction. Sci Pract Perspect 2005;3(1):4-10.

6. Volkow ND, Morales M. The brain on drugs: from reward to addiction. 2015; Cell 162:712-725.

7. Robison AJ, Nestler EJ. Transcriptional and epigenetic mechanisms of addiction. Nat Rev Neurosci 2011;12(11):623-637.

8. Buettner M, Toennes SW, Buettner S, et al. Nephropathy in illicit drug dbusers: a postmortem analysis. Am J Kidney Dis 2014;63(6):945-953.

9. Goel N, Pullman JM, Coco M. Cocaine and kidney injury: a kaleidoscope of pathology. Clin Kidney J 2014;7(6):513-517.
10. Jaffe J, Kimmel PL. Chronic nephropathies of cocaine and heroin abuse: a critical review. Clin J Am Soc Nephrol 2006;1: 655-667.

11. Nzrue CM, Hewan-Lowe K, Riley LJ Jr. Cocaine and the kidney: a synthesis of pathophysiologic and clinical perspectives. Am J Kidney Dis 2000;35(5):783-795.

12. Riezzo I, Fiore C, De Carlo D, et al. Side effects of cocaine abuse: multiorgan toxicity and pathological consequences. Curr Med Chem 2012;19:54624-5646.

13. Ersche KD, Doffinger R. Inflammation and infection in human cocaine addiction. Current Opinion in Behavioral Sciences 2017;13:203-209.

14. Van der Woude FJ. Cocaine use and kidney damage. Nephrol Dial Transplant 2000;15:299-301.

15. Richards JR, Garber D, Laurin EG, et al. Treatment of cocaine cardiovascular toxicity: a systematic review. Clin Toxicol (Phila) 2016;54(5):345-364.

16. Buydens-Branchey L, Branchey M. Association between low plasma levels of cholesterol and relapse in cocaine addicts. Psychosom Med 2003;65(1):86-91. 